\title{
Implicación De Las Empresas En La Formación Profesional Para EI Empleo. Análisis Sobre La Idoneidad De La Selección De Sus Acciones Formativas
}

\author{
Inmaculada Aznar-Díaz, (Dra.) \\ Universidad de Granada, España \\ Rafael Rey de la Cruz, (MSc.) \\ Centro Psicológico Carpe Diem, Córdoba, España \\ José María Romero-Rodríguez, (Prof.) \\ Universidad de Granada, España
}

Doi: 10.19044/esj.2018.v14n10p18 URL:http://dx.doi.org/10.19044/esj.2018.v14n10p18

\begin{abstract}
The purpose of this paper is to approach the development of Vocational Training for Employment in Spanish companies. The research carried out follows a descriptive methodology with which it evaluated the essential aspects for the selection of training actions by companies. Among the results we find that this selection of training can be improved and pedagogical and training factors must be prioritized over economic. In summary, the data lead us to an analysis and reflection on the reality that is presented in terms of Training for Employment in Spain.
\end{abstract}

Keywords: Continuing professional development, lifelong learning, employability, competence

Resumen

El trabajo tiene como objetivo la aproximación al desarrollo de la Formación Profesional para el Empleo en las empresas españolas. La investigación realizada sigue una metodología descriptiva con la que se evalúan los aspectos esenciales para la selección de las acciones formativas por parte de las empresas. Entre los resultados nos encontramos que dicha selección de la formación es mejorable y deben priorizarse factores pedagógicos y formativos sobre los económicos. En definitiva, los datos nos llevan a un análisis y reflexión sobre la realidad que se presenta en cuanto a Formación para el Empleo en España. 
Palabras clave: Formación profesional continua, aprendizaje permanente, empleabilidad, competencia.

\section{Introducción}

Hoy en día, la sociedad está inmersa en una situación compleja a la vez que cambiante, lo que requiere la continua actualización formativa de los trabajadores. Según el diccionario de la Real Academia (2014), el término formación hace referencia a la "acción o efecto de formar o formarse", siendo el término formar "adquirir una persona más o menos desarrollo, aptitud o habilidad en lo físico o en lo moral". En base a éste y coincidiendo con Casares (1990), en el concepto de formación encontramos un proceso superior a la mera instrucción, en la que la persona ha interiorizado esquemas conceptuales propios, poniendo en práctica razonamientos y principios que los hacen aplicables a la acción concreta.

La formación tal cual se entiende hoy, es explicada desde diversos conceptos situados muy próximos entre sí, sin los cuales sería muy difícil de comprender en la actualidad, entre ellos: sociedad del conocimiento, formación a lo largo de la vida o competencias profesionales. En pleno siglo XXI no cabe duda de que la sociedad en la que vivimos se encuentra basada en el conocimiento. La expresión "sociedad del conocimiento" se ha convertido en uno de los ejes centrales que explican y rodean muchas de las políticas socioeconómicas que se producen. Además este concepto lleva implícita la idea sobre la integración de las tecnologías de la información y comunicación (TIC) en el desarrollo de políticas educativas (Ornellas, Sánchez, Fraga y Domingo, 2015).

Acudiendo a la Organización de los Estados Americanos (2006), el tipo de sociedad necesario para competir y tener éxito frente a los cambios económicos y políticos del mundo moderno, hace referencia a la sociedad que está bien educada y que se basa en el conocimiento de sus ciudadanos para impulsar la innovación, el espíritu empresarial y el dinamismo de su economía.

Por tanto, se trata de un cambio en el sistema productivo mundial que obliga a la sociedad y en concreto a la actividad profesional de los formadores a una continua actualización, acceso al conocimiento $\mathrm{y}$, por consiguiente a un proceso conocido como "Lifelong learning" o formación a lo largo de la vida.

Todo ello conlleva una serie de repercusiones en el mercado laboral y la empleabilidad de las personas que quieren acceder a un puesto de trabajo. Es aquí donde entra en juego el término de competencia profesional, el cual, según el Ministerio de Educación Cultura y Deporte (1999), es el conjunto de conocimientos y capacidades que permiten el ejercicio de la actividad profesional conforme a las exigencias de la producción y el empleo. Es decir, en este entorno de una sociedad del conocimiento que exige una formación a 
lo largo de la vida (Aznar, Cáceres e Hinojo, 2011), las competencias profesionales son un requisito sine qua non para entrar y permanecer en el mercado laboral actual. Así, es como llegamos hasta el eje central de nuestra investigación, la Formación Profesional para el Empleo, entendida como proceso de adquisición de competencias profesionales que nos habiliten y mantengan en un puesto laboral.

Cabe resaltar que este trabajo forma parte de una investigación más amplia que deriva de un Trabajo Final de Grado con el título "Análisis de la formación profesional para el empleo", defendido en la Universidad de Granada en junio de 2015 para la obtención del Grado en Pedagogía (Rey, 2015).

\section{Marco conceptual de la Formación Profesional para el Empleo}

La Formación Profesional para el Empleo queda legislada en España en el R.D.395/2007, de 23 de marzo como:

Subsistema integrado por el conjunto de instrumentos y acciones que tienen por objeto impulsar y extender entre las empresas y los trabajadores ocupados y desempleados una formación que responda a sus necesidades y contribuya al desarrollo de una economía basada en el conocimiento ( $p$. 15584).

Los fines que persigue con las acciones que engloba la Formación Profesional para el Empleo según el R.D. 395/2007 son:

a) Favorecer la formación a lo largo de la vida de los trabajadores desempleados y ocupados, mejorando su capacitación profesional y desarrollo personal.

b) Proporcionar a los trabajadores los conocimientos y las prácticas adecuados a las competencias profesionales requeridas en el mercado de trabajo y a las necesidades de las empresas. empresas.

c) Contribuir a la mejora de la productividad y competitividad de las

d) Mejorar la empleabilidad de los trabajadores, especialmente de los que tienen mayores dificultades de mantenimiento del empleo o de inserción laboral.

e) Promover que las competencias profesionales adquiridas por los trabajadores tanto a través de procesos formativos (formales y no formales), como de la experiencia laboral, sean objeto de acreditación.

A su vez, ésta se encuentra integrada por:

a) La formación de demanda, que abarca las acciones formativas de las empresas y los permisos individuales de formación.

b) La formación de oferta, que comprende los planes de formación dirigidos prioritariamente a trabajadores ocupados y las acciones formativas dirigidas prioritariamente a trabajadores. 
c) La formación en alternancia con el empleo, permitiendo al trabajador compatibilizar la formación con la práctica profesional en el puesto de trabajo.

d) Las acciones de apoyo y acompañamiento a la formación, que son aquellas que permiten mejorar la eficacia del subsistema de formación profesional para el empleo.

\section{Gestión de la Formación Profesional para el Empleo}

En España, el sistema de Formación Profesional para el Empleo es gestionado por la Fundación Tripartita de la Formación para el Empleo (Fundación Tripartita, 2007). Ésta fundación es la encargada de velar y controlar todas aquellas políticas y acciones referentes a la formación profesional de los trabajadores del territorio nacional.

Ésta se encuentra englobada dentro del Ministerio de Empleo y Seguridad Social, concretamente a través del Servicio Público de Empleo Estatal (SEPE).

Como venimos detallando, es el R.D. 395/2007, en el que se sientan las bases de su desarrollo y por el que se rige. No obstante, con la llegada de la Ley 3/2012, de 6 de julio, se incluyeron aspectos esenciales como:

[...] el derecho del trabajador a 20 horas anuales de formación vinculadas al puesto de trabajo y el deber del empresario de formar a los trabajadores para adaptarlos a las modificaciones de dicho puesto, la cuenta de formación y el contrato para la formación que pretende mejorar la inserción laboral y la cualificación de los jóvenes combinando trabajo y formación ( $\mathrm{p}$. 49125).

\section{Metodología}

Los objetivos sobre los que se inciden en esta investigación son los siguientes:

Conocer la idoneidad de las elecciones que las empresas hacen de los cursos de formación.

- Identificar los criterios y su valor a la hora de seleccionar un curso de formación frente a otro.

- Concretar las características comunes de los cursos que las empresas llevan a cabo.

- $\quad$ Saber la opinión de los destinatarios sobre cómo la Administración controla la idoneidad y calidad de las acciones formativas.

- $\quad$ Conocer la percepción que los receptores (trabajadores) tienen de las acciones formativas.

El método de investigación escogido para realizar dicha investigación y atendiendo a los objetivos planteados, se basa en una metodología de corte descriptivo. 
Se han usado tanto técnicas cualitativas (entrevista semiestructurada) y cuantitativas (cuestionario), consultadas en Fernández (2006). Con ello estaremos dando mayor rigor y calidad a la investigación haciendo uso del complementarismo metodológico.

Esto es un aspecto esencial que nos va a permitir obtener una aproximación más cercana y acorde con la realidad. Tanto en éste como en cualquier proceso de investigación, se da la metáfora del iceberg, en la cual solo es visible la punta de dicho iceberg, no lo sumergido. Si solo nos quedamos con aquello que está a nuestra simple vista, la evaluación sería errónea.

Con la triangulación y confrontación de múltiples datos, técnicas y sujetos estaremos obteniendo variedad de evidencias que nos ayudarán a integrar mejor la información $\mathrm{y}$, por consiguiente a aproximarnos más fielmente a la realidad.

\section{Participantes}

Para llevar a cabo esta investigación se ha seleccionado un total de 18 sujetos, muestra que es representativa para afirmar los cambios y necesidades de formación de la organización objeto de estudio. Estos han sido escogidos en base a 2 categorías de actores en cuanto a la Formación para el Empleo:

a) Destinatarios de las acciones formativas (empleados que han cursado formaciones dentro de su empresa): 14 sujetos.

b) Personal de empresas (organizadores, impartidores, gestores... de la formación profesional para el empleo, en las propias empresas): 4 sujetos.

\section{Instrumentos de evaluación}

Se usan diversos instrumentos de evaluación en función principalmente de las categorías de actores anteriormente seleccionados como muestra.

Así, en la categoría a) Destinatarios de las acciones formativas, se usa el cuestionario. En la categoría b) Personal de empresas, se utiliza la entrevista semiestructurada. En cuanto al diseño de los instrumentos:

Para la realización del cuestionario nos hemos basado en las claves que aporta Martínez (2002), el cual desarrolla los aspectos a tener en cuenta a la hora de elaborar dicho instrumento. En este caso, hemos recurrido a los objetivos específicos anteriormente redactados para elaborar los ítems, ya que de este modo podremos recoger de manera precisa y concreta todo cuanto en esta investigación consideramos relevante.

Por otro lado, se ha diseñado una entrevista semiestructurada siguiendo los criterios de Anaya (2002). En función del grado de estructuración que se use en el instrumento de la entrevista tendremos un tipo u otro, ya que se acudió a los entrevistados con un guion previo, que nos permitió concretar e 
ir directamente a la información relevante, pero sin necesidad de tener un guion cerrado. El entrevistador por tanto debe ser el que guie de manera hábil y flexible al entrevistado, consiguiendo recoger la mayor información al respecto.

El motivo de esta elección de instrumento se debe a que las categorías de los sujetos que hemos seleccionado como muestra, son personas que poseen gran cantidad de información, experiencia y son grandes conocedores de la temática en cuestión, lo cual nos permite, conocer las particularidades y analizar al detalle nuestro problema de investigación.

\section{Resultados}

Los datos obtenidos a través de la aplicación del cuestionario a la muestra, nos proporciona los siguientes datos que analizamos y los más significativos pueden observarse en las figuras que se presentan. Se parte de que la totalidad de la muestra ha realizado alguna acción formativa (curso) organizada desde la empresa donde trabaja, considerando en el $67 \%$ de los casos que la duración de dichos cursos es la adecuada a los objetivos planteados en su programa (figura 1).

Figura 1. Duración acciones.

\section{¿Considera que la duración de la acción formativa fue la adecuada?}

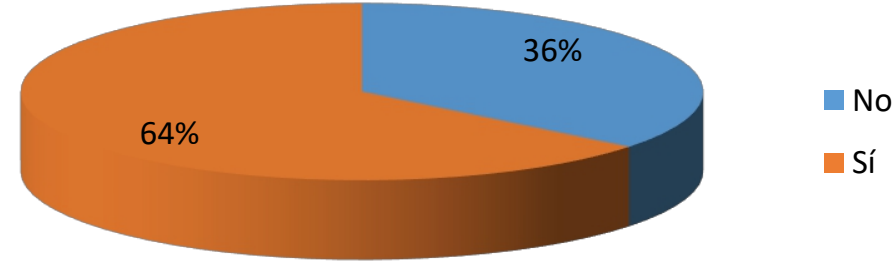

Respecto al objetivo específico que planteamos sobre los criterios de selección de un curso, buscando si existe la diversidad en la oferta formativa y si influye sobre todo la situación particular de cada empresa, la opinión de los destinatarios y no tanto la calidad de los mismos, en su elección, podemos observar como la opinión del empleado es tenida en cuenta mayoritariamente, con un $64 \%$ siendo preguntados directamente y con un $25 \%$ indirectamente (figura 2). En cambio, la realización de dicha formación es entendida por el $43 \%$ de los empleados como bastante necesaria, lo que indica que las acciones formativas son apreciadas por los empleados (figura 3 ). 
Figura 2. Opinión elección cursos.

\section{¿Cree que su opinión se tuvo en cuenta a la hora de escoger dicha acción formativa?}

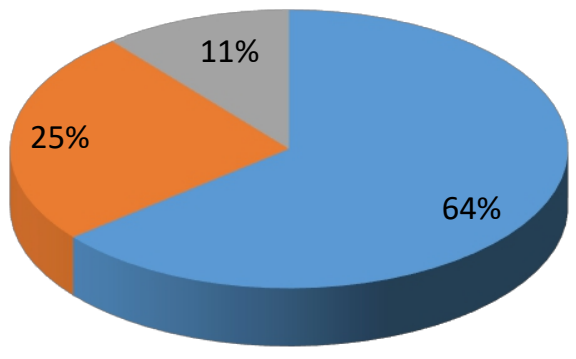

a) Sí, me preguntaron sobre qué necesitaba formarme

b) Sí, aunque no me preguntaron directamente

c) No tuvieron en cuenta la opinión de los destinatarios del curso

Figura 3. Necesidad de la formación.

\section{¿Piensa que necesitaba dicha formación?}

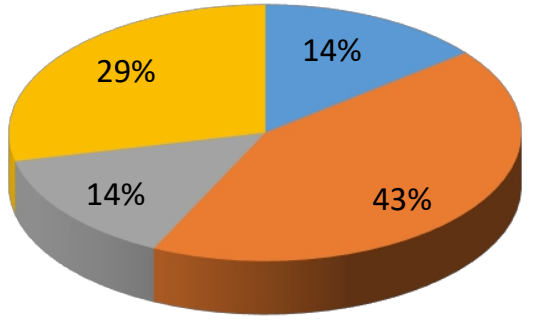

a) Mucho

b) Bastante

c) Poco

d) Nada

Sin embargo, el $43 \%$ de éstos perciben poco oportunos los criterios de selección, así como el 14\% nada, lo cual evidencia que la selección no es percibida por los destinatarios como la más oportuna. Ejemplo de ello es que a la pregunta ¿cree que la formación que recibió era la mejor?, el 55\% de los encuestados contestó que no, pensando que existen formaciones mejores para su situación que la que recibieron (figura 4). 
Figura 4. Formación mejor.

\section{¿Cree que la formación que recibió era la mejor?}

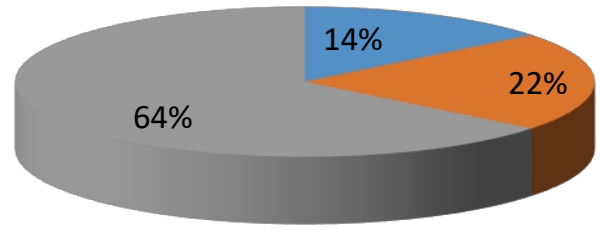

a) Sí, es sin duda la mejor

b) Normal, es una más de las muchas que hay.

c) No, creo que las hay mucho mejores

Concretamente, la calidad no es percibida por los encuestados como un factor relevante que en su empresa se haya tenido en cuenta a la hora de seleccionar los cursos. Solo el $23 \%$ la valora como buena, siendo mayoritaria la opción c) Normal, con un $46 \%$ (figura 5).

Figura 5. Calidad de la formación.

\section{¿Qué opinión tiene de la calidad de la formación recibida?}

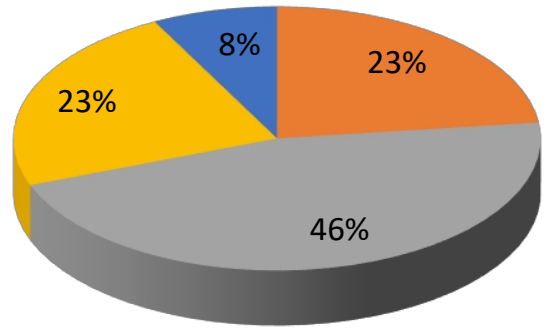

a) Excelente

b) Buena

c)Normal

d)Baja

e)Muy baja

Si contrastamos la opinión de los destinatarios con la del personal de empresas, encontramos que éstos últimos consideran la selección de los cursos como algo difícil, "no es fácil encontrar buenos cursos", en lo que influye la situación propia de la empresa como "el factor económico", "cumplir con el bono de formación, el tiempo...", las necesidades que detectan en los empleados, "en las áreas de mejora"...

Se puede observar como los entrevistados hacen poca referencia a la calidad en base a criterios pedagógicos y didácticos, ya que se centran en los factores anteriormente citados para justificar las selecciones que realizan. Esto 
es algo que puede explicar que los destinatarios valoren como mejorable la selección de las formaciones.

Por otro lado, el objetivo específico respecto a las características comunes de los cursos de formación que se ofrecen, destacan los cursos online y los cursos presenciales puntuales con ponentes o tutores especializados en la materia. Todos los entrevistados coinciden en que la tendencia actual de la formación para el empleo gira en torno a las acciones de teleformación, aunque siguen teniendo presencia y alta relevancia las formaciones presenciales con tutores especializados en la temática de dicha acción formativa.

Los datos obtenidos también revelan, respecto a otro de los objetivos específicos que el control de la formación que se realiza en las acciones formativas es escaso, poco efectivo. En este sentido, el $57 \%$ de los destinatarios de la formación no percibió control, frente al 29\% que lo percibió por la empresa y el 14\% por parte de la administración pública (figura 6). Y todo el personal de empresas entrevistado coincidió en que los controles son "livianos", "poco efectivos", "escasos"...

Nos parece anecdótico el hecho de que todos los entrevistados hiciesen referencia a los fraudes de formación acontecidos, lo que afianza la idea de que su control no ha sido, ni está siendo adecuado. Aun así, y relacionándolo con la presencia cada vez más notoria de los cursos e-learning, añaden que están favoreciendo el mayor control y seguimiento de las mismas.

Figura 6. Control de la calidad.

\section{¿Percibió control de la calidad e idoneidad de su formación?}

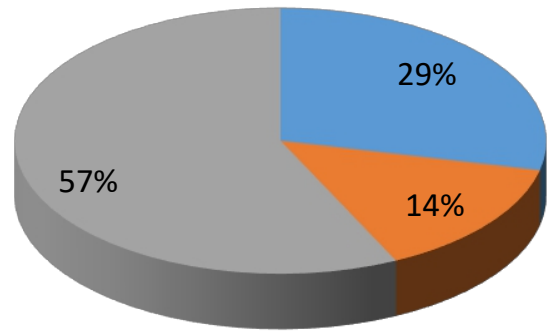

a)Sí, por parte de mi empresa

b) Sí por parte de la administración pública

Finalmente destacar que la percepción que se tiene de las acciones formativas es de aceptación, aunque evidencian que pueden ser mejores en cuanto a calidad y selección.

El 50\% de los encuestados las valoran al acabarlas con aceptación y el $29 \%$ con satisfacción y aprovechamiento (figura 7). Sin embargo, el 55\% de los encuestados coincide en que existen formaciones mucho mejores (figura 
4). Ejemplo de ello es que el $46 \%$ consideran la calidad de los cursos como normal, frente al $23 \%$ de buena (figura 5).

Figura 7. Satisfacción al finalizar.

\section{¿Qué impresión le causó al finalizarla?}

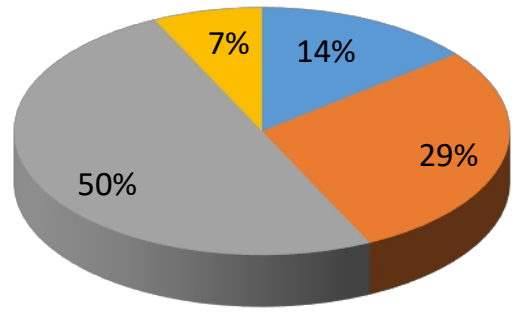

a) Indiferencia

b) Satisfacción y aprovechamiento

c) Aceptación

d) Rechazo

En la misma línea, los entrevistados evidencian que las formaciones son "normales, son formaciones un poco rutinarias", "no demasiado fuertes", "no es fácil encontrar buenos cursos". No obstante la satisfacción general que se tiene de esas acciones es $7 \%$ excelente, $14 \%$ buena, $57 \%$ normal (figura 8 ), lo que denota que están satisfechos pero no en alto grado.

Figura 8. Satisfacción en general.

\section{El grado de satisfacción general con la formación que ha recibido es...}

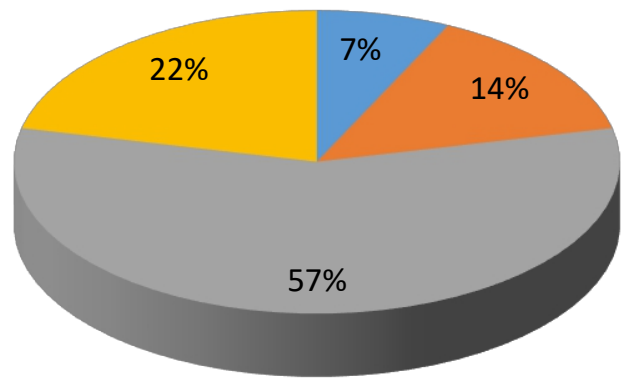

a) Excelente

b) Bueno

c) Normal

d) Bajo

En cuanto a los resultados de la entrevista semiestructurada, a continuación se recogen las respuestas más relevantes de los entrevistados para cada una de las 5 categorías, divididas en distintos indicadores. 


\section{Categoría. Opinión formación empresa:}

Indicador 1. Realización de la formación.

- $\quad$ Hacerse se hace... tú ya me entiendes...

- $\quad$ Pesa más el hacerla porque hay que hacerla que porque se necesite

Indicador 2. Dificultad de la formación.

- $\quad$ Normales, son formaciones un poco rutinarias.

- $\quad$ Muy especializada y práctica.

- $\quad$ Son muy light.

- No son demasiado fuertes.

Indicador 3. Satisfacción con la formación.

- Muchas de esas acciones no son ni las más idóneas, ni verdaderamente aportan mejoras.

- $\quad$ No nos salimos en exceso de 2 o 3 temáticas.

- Positiva. Aquí se prioriza mucho como base del éxito de la empresa.

Muchas de ellas no son ni productivas ni muy bien valoradas por los trabajadores.

\section{Categoría. Adecuación criterios pedagógicos:}

Indicador 1. Criterios en los que se basa la empresa.

Priman criterios como el prestigio de los ponentes, la empresa organizadora, si tienen parte práctica o no...

- De calidad pienso que sí... lo de los criterios pedagógicos o didácticos... tengo mis dudas, es evidente que algún que otro criterio de esos sí que habrá pero...

- $\quad$ Se basan en materiales, contenidos, docentes, plataformas y espacios de calidad alta.

- $\quad$ No es fácil encontrar buenos cursos específicos.

\section{Categoría. Selección de los cursos:}

Indicador 1. Opinión sobre los docentes.

- $\quad$ Aún podrían haber sido un poco mejores e innovadores.

- $\quad$ Un poco más creativos y trabajar los contenidos de un modo menos tradicional.

- $\quad$ Son muy rutinarios y clásicos.

Indicador 2. Opinión de los docentes.

- $\quad$ Siempre vamos con una idea muy fija de lo que queremos.

- $\quad$ Intentamos seguir nuestro plan de formación trazado en nuestra memoria anual, el cual parte de un análisis de necesidades, teniendo en cuenta las áreas de mejora.

- $\quad$ Pesa mucho el factor económico. 
Intentamos escoger cursos que sabemos que necesitamos.

\section{Categoría. Características comunes:}

Indicador 1. Modalidad de formación.

Hay mucha oferta online. Aunque muchas de esas plataformas son tan insufribles, tan ambiguas y... y... con tan poca claridad que pocas veces ayudan al aprendizaje.

- $\quad$ No hay mucho más salvo los cursos presenciales, los cuales siempre intentan ser impartidos con personas relevantes en la materia.

- $\quad$ Las resumiría en teleformaciones, con tutores relativamente especializados mezclado con formaciones presenciales muy concretas de formadores o ponentes de mayor prestigio que desarrollan una temática.

- $\quad$ La modalidad presencial, o lo que viene siendo las charlas formativas, son muy valoradas.

\section{Categoría. Control de la formación:}

Indicador 1. Tipo de controles.

Los controles no son demasiado efectivos, el limitarte a pasar una lista de asistencia y a entregar o no un determinado ejercicio no creo que asegure nada.

- $\quad$ No somos fraudulentos, pero sí que es cierto que los controles son muy livianos y se pueden falsear fácilmente.

Indicador 2. Percepción sobre el control formativo.

- $\quad$ Todo el mundo es consciente de la falta de controles que había sobre los cursos.

- $\quad$ El objetivo era gastar por gastar el bono y no el formarse.

- $\quad$ Yo percibo que es todo muy... muy escaso.

\section{Discusión y conclusion}

Como cierre de esta investigación se concluye que la Formación Profesional para el Empleo es una temática que requiere una continua reflexión y análisis, dentro del marco de una sociedad del conocimiento. Esto es lo que nos llevó a la realización de esta investigación, buscando dar a la Pedagogía el valor de requisito sine qua non en cuanto a la formación.

A partir de la revisión bibliográfica y a la investigación realizada, se ha profundizado y valorado la problemática planteada inicialmente acerca de si es correcta la elección de los cursos de formación profesional para el empleo que realizan las empresas desde el punto de vista pedagógico, hecha operativa en el objetivo del proyecto "conocer la idoneidad de las elecciones que las empresas hacen de los cursos de formación". 
En consonancia, la selección de los cursos es mejorable, ya que existen otros factores ajenos a los puramente formativos y pedagógicos que tienen gran relevancia a la hora de la selección. Esto no quiere decir que no sean tenidos en cuenta, sino que su importancia no se corresponde con el valor que verdaderamente tienen. Esto ocasiona como hemos podido observar, que las formaciones sean mediocres, comunes y rutinarias, no consiguen obtener el máximo provecho a algo tan fundamental y necesario en la situación que nos encontramos como es la formación continua. Por tanto, coincidiendo con los planteamientos de Tomás-Folch y Duran-Bellonch (2017) es necesario contextualizar los contenidos a la realidad de los participantes, así como fomentar una cultura de trabajo en equipo para asegurar una adecuada transferencia del campo teórico al práctico.

Los resultados nos arrojan que es necesaria una reformulación general del concepto e idea de formación, ya no solo por parte de los destinatarios entendidos como los trabajadores de una empresa, sino por las propias empresas que los organizan y diseñan, las administraciones públicas y, en definitiva, todos los agentes involucrados. Del mismo modo que como indican Jiménez, Sánchez y Sánchez (2010) se debe facilitar la asistencia de los trabajadores a las acciones formativas o implementar más acciones semipresenciales o e-learning.

La nueva reforma de la ley que se ha acontecido en el transcurso de la investigación es signo de esta necesidad, la cual esperemos que cambie y redefina en todos los implicados la concepción de la formación.

La formación es el motor de propulsión de la sociedad, personas capacitadas, competitivas y actualizadas son necesarias para superar los desafíos que nos aguardan en un futuro no muy lejano. No podemos considerarla como una obligación o un imperativo legislativo sino como una oportunidad de adentrarse y mantenerse en una sociedad en continuo cambio.

\section{References:}

1. Anaya, D. (2002). Diagnóstico en Educación. Diseño y uso de instrumentos. Madrid: Sanz y Torres.

2. Aznar Díaz, I., Cáceres Reche, M.P. y Hinojo Lucena, M.A. (2011). La formación profesional reglada en Andalucía a través de las percepciones de los equipos directivos desde una dimensión organizacional. Revista de Ciencias de la Educación del Instituto Calasanz, 228. 525-542.

3. Casares, P.M. (1990). Introducción a las ciencias de la educación. Granada: ICE.

4. Fernández, A. y Vallejo, M. (2006). Evaluación de programas, centros y profesores. Cuaderno de metodología. Granada: Grupo Editorial Universitario. 
5. Fundación Tripartita (2007). Formación para el empleo. Madrid: Fundación Tripartita. Recuperado de: http://www.fundaciontripartita.org/Con\%C3\%B3cenos/Pages/PForm aci\%C3\%B3nempleo.aspx

6. Jiménez, S., Sánchez, R. y Sánchez, G. (2010). Los institutos de administración pública en España: programas de formación para el personal al servicio de la administración. Estudios Gerenciales, 26(1), 169-192.

7. Martínez, F. (2002). El cuestionario. Un instrumento para la investigación en las ciencias sociales. Barcelona: Laertes Psicopedagogía.

8. Ministerio de Educación Cultura y Deporte (1999). Incual. Madrid: MECD. Recuperado de: http://www.mecd.gob.es/educa/incual/ice_faqs.html\#faq01

9. Organización de los Estados Americanos (2006). Sociedad del conocimiento. Santo Domingo: OAS. Recuperado de: http://www.oas.org/es/temas/sociedad_conocimiento.asp

10. Ornellas, A., Sánchez, J.A., Fraga, L. y Domingo, L. (2015). Políticas y prácticas en la formación permanente del profesorado en TIC en Cataluña. Revista Electrónica Interuniversitaria de Formación del Profesorado (REIFOP), 18(3), 83-96. doi:10.6018/reifop.18.3.190271

11. R.D. 395/2007, de 23 de marzo. En Boletín Oficial del Estado, núm. 87, de 11 de abril de 2007, por el que se regula el subsistema de formación profesional para el empleo.

12. Real Academia Española (2014). Diccionario de la lengua española (23a. ed.). Madrid: Espasa-Calpe.

13. Rey, R. (2015). Análisis de la formación profesional para el empleo. (Trabajo Final de Grado). Granada: Facultad de Ciencias de la Educación, Universidad de Granada. Recuperado de http://digibug.ugr.es/bitstream/10481/41739/1/Rey_de_la_Cruz\%2c_ Rafael.pdf

14. Tomás-Folch, M. y Duran-Bellonch, M. (2017). Comprendiendo los factores que afectan la transferencia de la formación permanente del profesorado. Propuestas de mejora. Revista Electrónica Interuniversitaria de Formación del Profesorado (REIFOP), 20(1), 145-157. doi:10.6018/reifop.20.1.240591 